\title{
An extension of the physical theory of diffraction concept for aperture radiation problems
}

\author{
Ayhan Altıntaş \\ Department of Electrical and Electronics Engineering, Bilkent University, Ankara, Turkey \\ O. Merih Büyükdura \\ Department of Electrical and Electronics Engineering, Middle East Technical University, Ankara, Turkey
}

Prabhakar H. Pathak

ElectroScience Laboratory, The Ohio State University, Columbus

\begin{abstract}
A correction to the Kirchhoff-Huygens approximation in the format of a diffraction coefficient is derived for an aperture terminated by a half plane. As in the physical theory of diffraction (PTD), this is achieved by considering the end point contribution to the aperture integral. It is seen that when the aperture is taken as conformal with the surface of the half plane, the conventional PTD result is obtained.
\end{abstract}

\section{Introduction}

The physical theory of diffraction (PTD) [Ufimtsev, $1962]$ is widely used in predicting the scattering from conducting bodies containing edges. However, in some problems such as the modal transmission through the junction of two waveguide sections or the radiation from an open-ended waveguide, the conventional PTD cannot be used. In this case, one usually resorts to analysis via aperture integration. One widely used approximation is the Kirchhoff-Huygens (KH) approximation in which the fields at an aperture are taken as the geometrical optics (GO) fields. Although the asymptotic evaluation of this integral gives some end point contributions to account for edge diffraction effects, they are not as accurate as those predicted by the geometrical theory of diffraction (GTD) [Keller, 1962] analysis [Altıntaş, 1986; Jull, 1973]. A proper correction to the edge effects predicted by the $\mathrm{KH}$ approximation is given by Michaeli [1985], but for the purpose of obtaining closed-form results for doublediffraction problems. The PTD involves a correction to the physical optics (PO) approximation in that a diffractionlike correction term is added to the PO radiation integral. We propose to introduce a similar diffractionlike

\section{Copyright 1994 by the American Geophysical Union.}

Paper number 94RS01667.

0048-6604/94/94RS-01667\$08.00 correction to the $\mathrm{KH}$ aperture integration approximation. It is noted that the aperture integration is still to be taken exactly or numerically and the diffractionlike correction is added to it at the edges as in the PTD. The canonical problem considered for this purpose is the plane wave scattering from a perfectly conducting half plane.

The PTD procedure for a two-dimensional problem is briefly described in section 2 , where the present method is also formulated. Numerical examples are given and discussed in section 3. Finally, concluding remarks appear in section 4. An $e^{j \omega t}$ time variation for the fields is assumed and suppressed in the following development.

\section{Analysis}

Consider the perfectly conducting half plane located in the region $y=0, x>0$ as shown in Figure 1. The structure is illuminated by a plane wave incident from the $\phi^{\prime}$ direction, and the field at $(\rho, \phi)$ is of interest. The problem is scalar in that the field $u(\rho, \phi)$ represents the $z$ component of either the electric or magnetic field. The former case is as usual directly related to the acoustic "soft" case and is denoted by the subscript $s$, whereas the latter is called the acoustic "hard" case and denoted by $h$. The incident field is given by

$$
u^{i}=u_{0} e^{j k \rho \cos \left(\phi-\phi^{\prime}\right)} .
$$




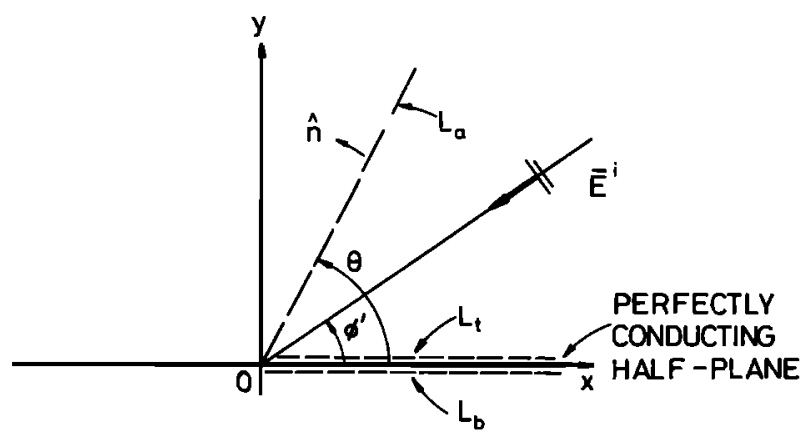

Figure 1. Perfectly conducting half plane.

The PTD approximation to the scattered field is given by

$$
\begin{aligned}
u_{s, h}^{P T D}= & \int_{L_{t}}\left[u^{G O}\left(\vec{\rho}^{\prime}\right) \frac{\partial G_{0}\left(\vec{\rho}, \vec{\rho}^{\prime}\right)}{\partial n^{\prime}}-G_{0}\left(\vec{\rho}, \vec{\rho}^{\prime}\right)\right. \\
& \left.\frac{\partial u^{G O}\left(\vec{\rho}^{\prime}\right)}{\partial n^{\prime}}\right] d l^{\prime}+u^{i}(0) D_{s, h}^{u} \frac{e^{-j k \rho}}{\sqrt{\rho}}
\end{aligned}
$$

where $L_{t}$ denotes the top surface of the half plane, $\vec{n}^{\prime}$ is the unit normal to the half plane, and

$$
G_{0}\left(\rho, \phi \mid \rho^{\prime}, \phi^{\prime}\right)=-\frac{j}{4} H_{0}^{(2)}\left(k\left|\vec{\rho}-\vec{\rho}^{\prime}\right|\right)
$$

is the two-dimensional (2-D) free-space Green's function. Note that $u$ and $\partial u / \partial n^{\prime}$ in the integral of (2) have been replaced by their $\mathrm{GO}$ approximations and they are given explicitly by

$$
u^{G O}= \begin{cases}2 u^{i} & \text { for the hard case } \\ 0 & \text { for the soft case }\end{cases}
$$

and

$$
\frac{\partial u^{G O}}{\partial n^{\prime}}= \begin{cases}0 & \text { for the hard case } \\ 2\left(\partial u^{i} / \partial n^{\prime}\right) & \text { for the soft case. }\end{cases}
$$

The diffraction coefficent, $D_{s, h}^{u}$, appearing in (2) is given by [Pathak, 1988; Lee, 1977]

$$
D_{s, h}^{u}\left(\phi, \phi^{\prime}\right)=D_{s, h}^{G T D}\left(\phi, \phi^{\prime}\right)-D_{s, h}^{P O}\left(\phi, \phi^{\prime}\right),
$$

and it is referred to as the Ufimtsev diffraction correction coefficient. $D_{s, h}^{G T D}\left(\phi, \phi^{\prime}\right)$ is the GTD edge diffraction coefficient, and $D_{s, h}^{P O}\left(\phi, \phi^{\prime}\right)$ is the PO diffraction coefficient obtained from the asymptotic end point contribution to the PO integral appearing in (2). The $D_{s, h}^{u}\left(\phi, \phi^{\prime}\right)$ is given explicitly by

$$
\begin{aligned}
D_{s, h}^{u}\left(\phi, \phi^{\prime}\right)= & -\frac{e^{-j \frac{\pi}{4}}}{2 \sqrt{2 \pi k}}\left[\sec \frac{\phi-\phi^{\prime}}{2} \mp \sec \frac{\phi+\phi^{\prime}}{2}\right. \\
& \left.-\tan \frac{\phi-\phi^{\prime}}{2} \pm \tan \frac{\phi+\phi^{\prime}}{2}\right],
\end{aligned}
$$

where the upper (lower) sign corresponds to the soft (hard) case. One important property of the Ufimtsev or PTD diffraction coefficients is that unlike the GTD diffraction coefficients, they can be employed for observation points in the transition regions of the GO shadow boundaries. This is due to the fact that the singularities of $D_{s, h}^{G T D}$ are cancelled by those of $D_{s, h}^{P O}$ along these boundaries. We shall now introduce a similar correction to the $\mathrm{KH}$ approximation.

Let $L_{b}$ denote the bottom (dark) surface of the half plane, and $L_{a}$ denote an aperture surface making an angle $\theta<\phi^{\prime}+\pi$ with $L_{t}$ as shown in Figure 1. The KH approximation to the scattered field is given by

$$
\begin{aligned}
u^{K H}= & \int_{L_{a}}\left[u^{G O}\left(\rho^{\prime}, \theta\right) \frac{\partial G_{0}\left(\rho, \theta ; \rho^{\prime}, \theta\right)}{\partial n}\right. \\
& \left.-G_{0}\left(\rho, \theta ; \rho^{\prime}, \theta\right) \frac{\partial u^{G O}\left(\rho^{\prime}, \theta\right)}{\partial n}\right] d l^{\prime},
\end{aligned}
$$

where the contribution from $L_{b}$ vanishes, since $u^{G O}=$ $\partial u^{G O} / \partial n=0$ there, and $\vec{n}$ is shown in the figure. This approximation is valid only in the region $\phi>\theta$. The GO approximation to the field at the aperture $L_{a}$ is given by

$$
\begin{aligned}
u^{G O}= & u(0) e^{j k x \cos \phi^{\prime}+j k y \sin \phi^{\prime}} \\
& \pm e^{j k x \cos \phi^{\prime}-j k y \sin \phi^{\prime}} U\left(\pi-\phi^{\prime}-\theta\right),
\end{aligned}
$$

where the unit step function $U$ (with $U(\xi)=1$, if $\xi>0$, and $U(\xi)=0$, if $\xi<0$ ) has been introduced to account for whether or not the aperture is illuminated by the reflected field. The asymptotic evaluation of the integral in (8)

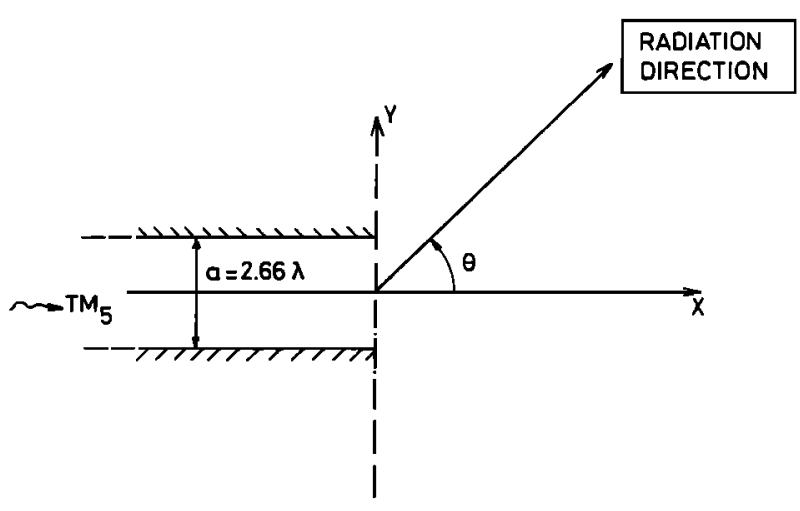

Figure 2. Open-ended parallel plate waveguide. 


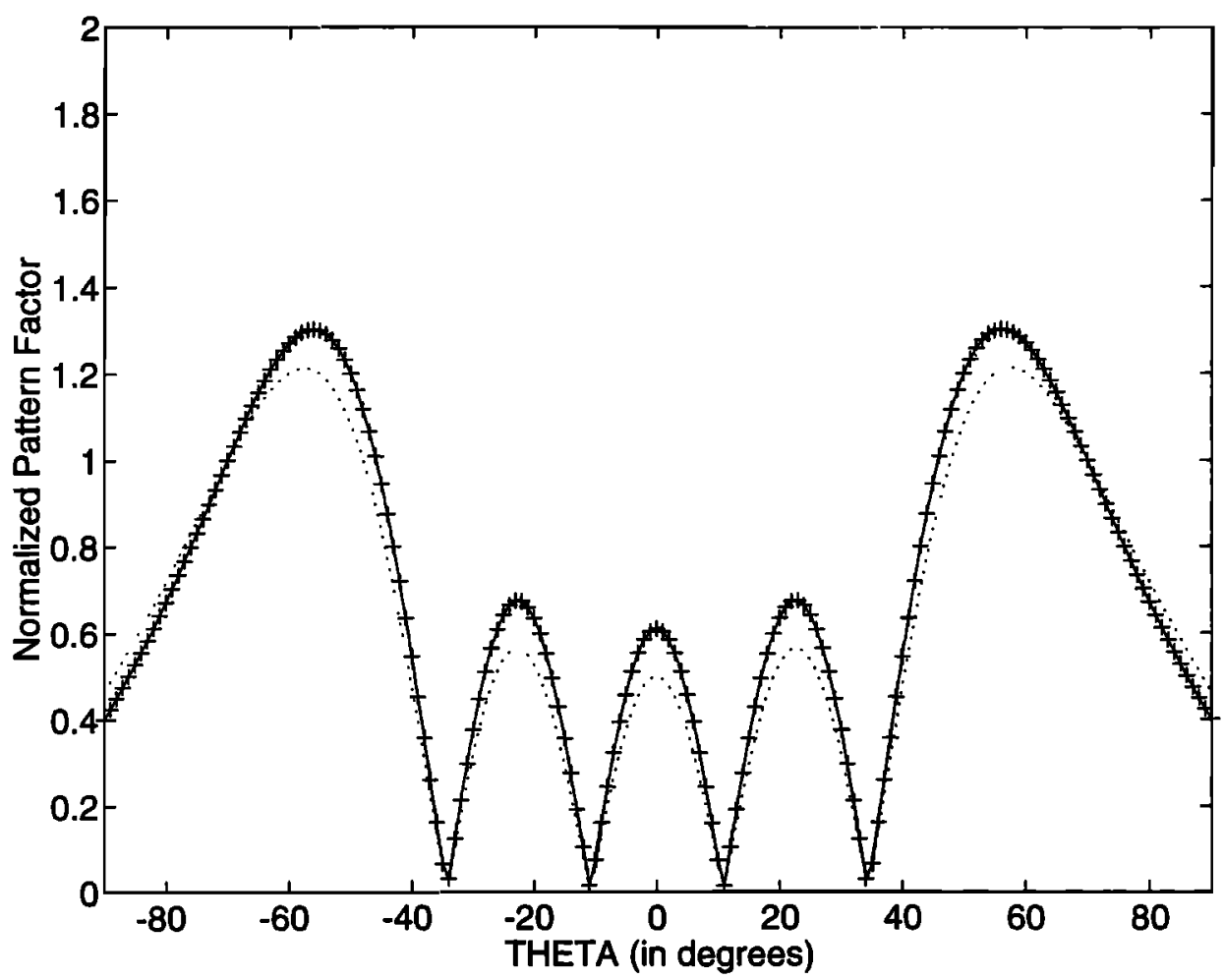

Figure 3. $T M_{5}$ mode radiation from the open-ended parallel plate waveguide of Figure 2; dotted line, KH approximation; crosses, GTD; solid line, present method.

yields the GO field and an end point contribution, the latter of which is in the form of an edge-diffracted field

$$
u_{d}^{K H}=u^{i}(0) D_{s, h}^{K H}\left(\phi, \phi^{\prime}\right) \frac{e^{-j k \rho}}{\sqrt{\rho}},
$$

where

$$
\begin{aligned}
D_{s, h}^{K H}= & -\frac{e^{-j \frac{\pi}{4}}}{2 \sqrt{2 \pi k}}\left[\tan \frac{\phi-\phi^{\prime}}{2}\right. \\
& \left.\mp \tan \frac{\phi+\phi^{\prime}}{2} U\left(\pi-\phi^{\prime}-\theta\right)\right] .
\end{aligned}
$$

It is obvious from (11) that when the aperture is illuminated by both the incident and the reflected fields, then $\theta<\pi-\phi^{\prime}$, and $D_{s, h}^{K H}=D_{s, h}^{P O}$. A correction to the $\mathrm{KH}$ appproximation similar to the PTD correction is therefore given by

$$
\begin{aligned}
u^{K T D}= & \int_{L_{a}}\left[u^{G O}\left(\vec{\rho}^{\prime}\right) \frac{\partial G_{0}\left(\vec{\rho}, \vec{\rho}^{\prime}\right)}{\partial n^{\prime}}\right. \\
& \left.-G_{0}\left(\vec{\rho}, \vec{\rho}^{\prime}\right) \frac{\partial u^{G O}\left(\vec{\rho}^{\prime}\right)}{\partial n^{\prime}}\right] d l^{\prime} \\
& +u^{i}(0) D_{s, h}^{K T D}\left(\phi, \phi^{\prime}\right) \frac{e^{-j k \rho}}{\sqrt{\rho}}
\end{aligned}
$$

where

$$
D_{s, h}^{K T D}\left(\phi, \phi^{\prime}\right)=D_{s, h}^{G T D}\left(\phi, \phi^{\prime}\right)-D_{s, h}^{K H}\left(\phi, \phi^{\prime}\right) .
$$

Note also that if $\theta<\pi-\phi^{\prime}$, then $D_{s, h}^{K T D}=D_{s, h}^{u}$. From (13), it is seen that the diffraction coefficients $D_{s, h}^{K T D}$ remain finite at the incident and reflection shadow boundaries. It is interesting that except for the discontinuity due to the inclusion (or discarding) of a term at the re-

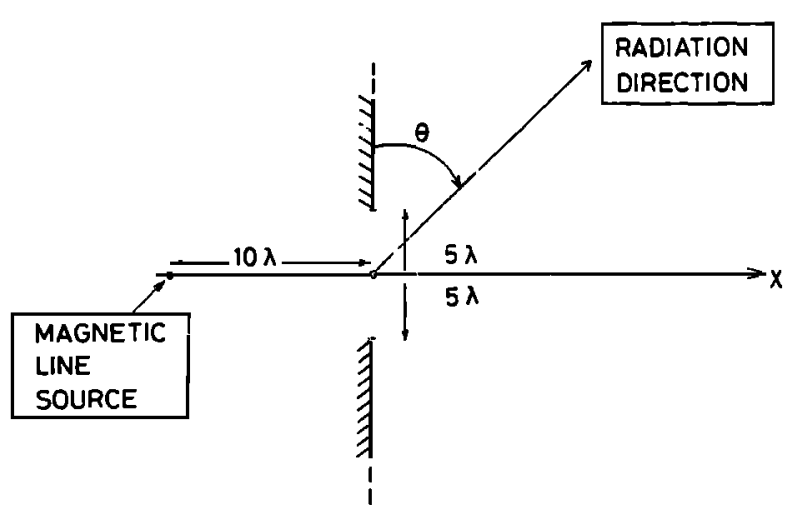

Figure 4. Magnetic line source and perfectly conducting screen. 
flection boundary $\theta=\pi-\phi^{\prime}$, the result is independent of $\theta$. But since the case $\theta=0$ yields precisely the PTD approximation, the PTD is a special case of the present procedure (KTD). The above mentioned abrupt change in the diffraction coefficient at $\theta=\pi-\phi^{\prime}$ is hardly surprising, considering that the GO field illuminating the aperture is discontinuous at this value of $\theta$. It is important to note that when the unit step function in (11) vanishes, the coefficient in (13) is not finite at the reflection boundary; however, in this case, $\theta>\pi-\phi^{\prime}$, and therefore the reflection boundary is not within the observation region of interest.

Extending the result in (13) to deal with a perfectly conducting wedge, rather than just the half plane, one obtains

$$
\begin{aligned}
D_{s, h}^{K T D}= & -\frac{e^{-3 \frac{\pi}{4}} \sin \frac{\pi}{n}}{n \sqrt{2 \pi k}}\left[\frac{1}{\cos \frac{\pi}{n}-\cos \frac{\phi-\phi^{\prime}}{n}}\right. \\
& \left.-\frac{1}{\cos \frac{\pi}{n}-\cos \frac{\phi+\phi^{\prime}}{n}}\right]-D_{s, h}^{K H}\left(\phi, \phi^{\prime}\right),
\end{aligned}
$$

where $n \pi$ is the exterior wedge angle. This result coincides with (27) of Michaeli [1985], except that the latter is restricted to the case where the aperture is not illuminated by the reflected field. Note that the PO integral, hence the PTD corection, involves only an electric type current density (one of the terms in (2) vanishes; see (4) and (5)), whereas the KH integral includes terms due to both electric and magnetic equivalent current densities.

\section{Numerical Results}

Consider the open-ended parallel plate waveguide shown in Figure 2 with a plate separation of $2.66 \lambda$. The field of the $T M_{5}$ mode is incident from the interior of the guide. The modal rays [Altıntaş et al., 1988] make an angle of about $70^{\circ}$ with the walls. The modal radiation patterns based on the KH approximation, GTD, and the present method are compared in Figure 3. Note that in this example the aperture is illuminated by both the incident and reflected modal rays. The results indicate that the present method improves the $\mathrm{KH}$ approximation so that it becomes indistinguishable from the GTD result, which is known to be accurate for this problem.

The second example is that of a magnetic line source radiating in the presence of a perfectly conducting screen with an aperture as shown in Figure 4. The field in the region $x>0$ is of interest. The far-zone radiation pattern is obtained by using the three methods mentioned above, and the results are shown in Figure 5. Note that the GTD result becomes singular, or it blows up at the shadow boundaries as expected and is known to be accurate away from these boundaries. The $\mathrm{KH}$ method is very accurate

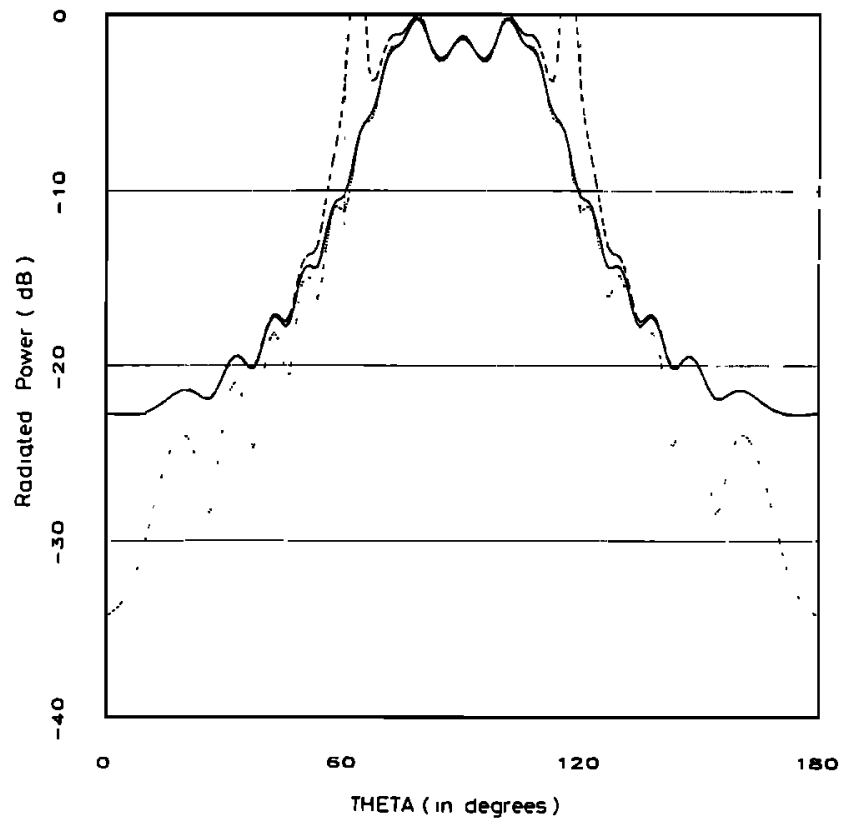

Figure 5. Magnetic line source radiation through the screen of Figure 4; dotted line, KH approximation; dashed line, GTD; solid line, present method.

near the main beam direction, but it gets less accurate far from the main beam direction. The result obtained by using the present method agrees with the other two where they are most accurate. In this example, the aperture is illuminated only by the incident field; hence the unit step function appearing in (11) (also implicitly in (13)) is taken as zero.

\section{Conclusion}

A diffraction correction to the $\mathrm{KH}$ approximation has been derived. It is seen that this $\mathrm{KH}$ correction diffraction coefficient has two different forms depending on the presence of the reflected field at the aperture chosen. An example for each case has been studied, and the results of the present method have been compared with those of the conventional GTD and the KH approximation. It has been observed that our method agrees with the other two approximations in their respective regions of accuracy. It is also noted that, unlike the GTD, the present method is free from singularities at shadow boundaries. Finally, we note that in the special case of choosing the aperture conformal with the conducting surface, our method reduces to the conventional PTD.

Acknowledgments. This work is partially supported by NATO's Scientific Affairs Division in the framework of the Science for Stability program. The useful comments by the reviewer(s) are also appreciated. 


\section{References}

Altıntaş, A., Electromagnetic scattering from a class of open-ended waveguide discontinuities, Ph.D. dissertation, Dept. of Elect. Eng., Ohio State Univ., Columbus, 1986.

Altıntas, A., M. C. Liang, and P. H. Pathak, A selective modal scheme for the analysis of EM coupling into or radiation from large open-ended waveguides, IEEE Trans. Antennas Propag., AP-36(1), 84-96, 1988.

Jull, E. V., Aperture fields and gain of open-ended parallel-plate waveguide, IEEE Trans. Antennas Propag., AP-2I(1), 14-18, 1973.

Keller, J. B., Geometrical theory of diffraction, J. Opt. Soc. Am., 52(2), 116-130, 1962.

Lee, S. W., Comparison of uniform asymptotic theory and Ufimtsev's theory of EM edge diffraction, IEEE Trans. Antennas Propag., AP. 25(2), 1977.

Michaeli, A., A new asymptotic high-frequency analysis of electromagnetic scattering by a pair of parallel wedges: Closed form results," Radio Sci., 20(6), 1537-1548, 1985.
Pathak, P. H., Techniques for high frequency problems, in Antenna Handbook, edited by Y. T. Lo and S. W. Lee, chap. A4, Van Nostrand Reinhold, New York, 1988.

Ufimtsev, P. Y., Method of edge waves in the physical theory of diffraction, Izd-Vo Sov. Radio, 1-243, 1962, translated and available from the U.S. Air Force Foreign Technology Division, Wright-Patterson Air Force Base, Ohio, 1971.

A. Altuntas, Department of Electrical and Electronics Engineering, Bilkent University, Bilkent, 06533 Ankara, Turkey. (e-mail: altintas@ee.bilkent.edu.tr)

O. M. Büyükdura, Department of Electrical and Electronics Engineering, 06531 Middle East Technical University, 06531 Ankara, Turkey.

P. H. Pathak, ElectroScience Laboratory, Ohio State University, 1320 Kinnear Rd., Columbus, Ohio 43212

(Received January 27, 1994; revised June 23, 1994;

accepted June 23, 1994.) 